\title{
Untangling the Complexity of the Valence of Actor Engagement: Conceptual Foundations, Propositions and Research Directions: An Abstract
}

\author{
Loic Pengtao Li, Biljana Juric, and Roderick J. Brodie
}

\begin{abstract}
The paper investigates the complexity associated with the valence of actor engagement in the service network and develops a research agenda for future studies in this area. The psychological foundations of valence are explored, and studies on the valence of engagement from the marketing discipline are analysed in order to provide more insights into the nature of the valence of actor engagement. This leads to the development of five sets of propositions that define the conceptual domain of the valence of actor engagement and provide a future research agenda in the area. The paper integrates different conceptual approaches to the valence of actor engagement in the service network. In particular, the study identifies various factors that influence an actor's positive and negative psychological dispositions and discusses engagement ambivalence, which results from the combination of positive and negative dispositions. The paper addresses the relationship between service network factors and the valence of actor engagement and engagement behaviours. We provide a comprehensive understanding of the valence of engagement in actor networks by taking into account different dimensions that define the conceptual domain, which leads to an agenda for future research. We conceptualise the valence of engagement in actor networks. In doing so, we recognise actor engagement ambivalence resulting from different positive and negative actor dispositions.
\end{abstract}

L.P. Li $(\bowtie) \bullet$ B. Juric • R.J. Brodie

University of Auckland, Auckland, New Zealand

e-mail: pengtao.li@auckland.ac.nz; b.juric@ auckland.ac.nz; r.brodie@ auckland.ac.nz 